\title{
A comparative analysis of in-office vital $6 \%$ hydrogen peroxide activated charcoal tooth whitening treatment enhanced with an 810nm diode laser, compared to 35\% hydrogen peroxide bleaching
}

SADJ March 2021, Vol. 76 No. 2 p64 - p71

SI Hassim¹, TA Muslim²

\section{ABSTRACT}

\section{Background}

Laser dental bleaching is considered to be a contemporary approach to enhancing the in-office power bleaching procedure.

\section{Objective}

To investigate if laser enhanced 6\% Hydrogen Peroxide (HP) solution is equivalent to $35 \% \mathrm{HP}$ solution over a two-visit power bleaching treatment protocol.

\section{Materials and methods}

In a randomised double-blinded clinical trial, 43 patients were assigned to a group that received either the laser -enhanced $6 \%$ Hydrogen Peroxide $(n=21)$ treatment, or the standard 35\% Hydrogen Peroxide $(n=22)$ treatment, over two visits, with a one-week interval. Activated charcoal HP paste was prepared for both groups. The laser enhanced $6 \% \mathrm{HP}$ group received a dosage of $90 \mathrm{~J} / \mathrm{cm}^{2}$ per bleaching cycle using $810 \mathrm{~nm}$ diode laser.

Tooth colour was measured at the beginning and end of each session registering parameters $L^{*}, a^{*}$ and $b^{*}$, and tooth sensitivity. The calculated difference between these

\section{Author affiliations:}

1. Shabeer I Hassim: BDS (Medunsa), MSc (Lasers in Dentistry) (Rheinisch-Westfälische Technische Hochschule Aachen), Private Dentist, Durban, South Africa.

ORCID Number: 0000-0002-5559-7188

2. Tufayl A Muslim: BDentTher (UDW), MSc. [DENT] (UWC), MTech (Qual) (DUT), PhD (UWC), PG Dip Higher Education (UKZN), PG Dip Law (UKZN), Acting Academic Leader and Lecturer, Discipline of Dentistry, School of Health Sciences, University of KwaZuluNatal, Westville Campus, University Road, Durban, South Africa. ORCID Number: 0000-0001-5824-6191

Corresponding author: Shabeer I Hassim

Private Dentist, 35 Waverton Road,

Essenwood, Durban, 4001, KZN, South Africa.

Email: docshabz@aol.com

Author contributions:

1. Shabeer I Hassim: Principal Researcher, write up - $85 \%$

2. Tufayl A Muslim: Review and revision of write up - 15\% parameters, $\Delta E$, was the primary data focus. The mean $\triangle \mathrm{E}$ over the treatment duration was used to answer the research question by a t-test to evaluate group differences at $5 \%$ significance level.

\section{Results}

The analysis revealed that the null hypothesis could not be rejected and the results were inconclusive.

\section{Conclusion}

The observations expound the idea of an absorption enhancement mechanism, rather than a free radical activation, as the technique for improving bleaching outcomes.

\section{INTRODUCTION}

Dental bleaching is one of the eminently popular and safest cosmetic procedures available. With an increase in the demand for this procedure comes an increased need for this treatment to be administered efficaciously.

Dental bleaching is the process of altering the natural colour or shade of teeth, rendering them whiter and aesthetically improved in visual colour perception. Dental bleaching is differentiated from tooth whitening which covers a broader spectrum of which bleaching forms an aspect (for example, whitening toothpaste whiten teeth but do not bleach them).

In terms of patients' rationalisation, in-office dental bleaching treatments represent a plausibly convenient way to achieve an optimal bleaching result. The panacea of an instant tooth whitening seems alluring enough for those seeking whiter teeth. The modern patient expects an immediately acceptable, perceived aesthetic improvement in tooth colour as a determinant of value for both monetary and time inputs.

With higher concentrations of hydrogen peroxide at 35\% in-office bleaching has been found to be effective but may frequently require multiple visits to produce an opti- 
mal result. ${ }^{1}$ Clinicians tend to use a combination of home and office treatments to meet patients' expectations while avoiding the costs of additional chair time associated with multiple in-office treatments.

Laser optimisation of the in-office power bleaching treatment is a contemporary approach to accelerate peroxide bleaching that basis itself predominantly on the physical means of heat transfer to the bleach. Laser-tissue interactions warrant careful consideration so as not to cause tissue damage and unwanted side effects yet optimally improve bleaching efficacy.

\section{BACKGROUND AND LITERATURE REVIEW}

Hydrogen peroxide (HP) has been used as a dental bleach since $1884 .^{2}$ Its use, efficacy and safety as suitable dental bleaching agents have been well established. ${ }^{3}$ Application of HP as a bleaching agent occurs in a variety of formats [over-the-counter products, dentist-administered takehome products, and dentist-administered in-surgery whitening (power bleaching)]. The power bleaching technique aims to provide a maximum bleaching result in a relatively short space of time (the duration of a dental appointment).

Bowles and Ugwuneri ${ }^{4}$ designed an in vitro model able to detect the presence of HP in the pulp of bleached teeth. They concluded that dental hard tissues exhibit substantial permeability to HP and that this permeability increases with increasing temperature. ${ }^{5}$ Recently, MicroRaman spectroscopy (MRS) and Fourier transform infrared photoacoustic spectroscopy (FTIR-PAS) were used to measure spectra of specimens. MRS showed that HP crossed enamel had a marked concentration at the dentine-enamel junction (DEJ) and accumulated in dentine. ${ }^{6}$

FTIR-PAS showed that HP modified dentine's organic compounds demonstrated by a decrease in amides I, II, III absorption band intensities". ${ }^{6}$ This study showed a relationship between HP diffusion and oxidation of dentine organic components. Other studies have substantiated oxidation of organic components rather than the previous chromophore theory as the predominant mechanism in tooth bleaching.

A 2018 study concluded that dentine phosphoprotein (DPP), the main non-collagenous protein, was responsible for the fluorescence and colour of normal dentine. ${ }^{7}$ Moreover, HP might whiten normal dentine by oxidising DPP - specifically, the aromatic amino acids (AAAs) in DPP. ${ }^{7}$ Oxidation of organic components of dentine is concluded to be the predominant theory of the microscopic mechanism of tooth bleaching.

Enamel and dentine behave like semi-permeable membranes, and as such, follow the description of diffusion of HP by Fick's Second Law. 8,9 According to Fick's law, flux, or the rate HP will move through enamel and dentine, is equal to the gradient (pressure differential/thickness) multiplied by the diffusion constant.

Fick's law reveals that in diffusive processes there is a fundamental relationship with the time of HP application as well as the concentration of HP solution. It is essential to understand this relation as it is vital in correlating effi- cacy of HP in bleaching models. The efficacy of the use of high concentration HP gels, typically at $37 \%$, is well established in power bleaching techniques; the higher the concentration of the bleaching solution, the quicker a shade change will occur. ${ }^{10}$

The relationship between peroxide penetration and efficacy cannot be linearly correlated, as the system is complex with inter-dependable factors. Increased penetration, as well as efficacy, was noted for increased concentration $^{1,11}$ and time $e^{9,12,13}$ Torres et al., have shown that chemical activation resulted in decreased penetration but enhanced efficacy. ${ }^{1}$ Increased $\mathrm{pH}$ did not improve efficacy, ${ }^{15}$ but etching enamel with $37 \%$ phosphoric acid improved HP penetration. ${ }^{16}$ There was both improved efficacies as well as HP penetration in younger teeth with larger dentinal tubules. ${ }^{17,18}$

The addition of heat or light may speed up the catalytic reaction as well as improve the diffusion of HP. Other studies have noted Increased HP penetration for heat as well as laser activation. ${ }^{4,7,12}$ The dependence of the rate of a chemical reaction on temperature can be explained by the Arrhenius equation which states that as an increase in temperature occurs, then an increase in the fraction of molecules that have kinetic energies higher than the activation energy of the reaction occurs.

Therefore, the total activation energy of the reaction decreases, and as a result the rate of reaction increases. The addition of heat and use of lights and laser have not comprehensively been shown to enhance the bleaching effect or efficacy. ${ }^{20,21}$

A protocol of creating a sealed environment during inoffice bleaching, with claims of increased efficacy, called the "compressive bleaching technique" has been introduced, efficacy. ${ }^{22}$ Here the power-bleaching gel is applied using a bleaching tray and sealing the borders with a light-cured resin barrier.

Studies using a similar sealed technique with low concentration HP show both $\triangle \mathrm{E}$ and shade score changes were higher for the sealed bleaching group than the conventional bleaching group. Therefore, the effectiveness of the in-office bleaching protocol increased when the sealed bleaching technique was used. ${ }^{23}$ In-vitro analysis of sealed bleaching technique using high concentration HP showed increased penetration of HP but similar efficacy in terms of colour change. ${ }^{24}$

Currently, there are limited studies that compare or evaluate the efficacy of a $6 \% \mathrm{HP}$ solution. In a study using a concentration of $6 \% \mathrm{HP}$, patients achieved a change of at least 5 units delta $E(\Delta E)$ (a quantitative colourimetric calculation difference from spectrometer measurements taken before and after treatment) in a 9-month follow-up which was considered effective. ${ }^{25}$

In a split-mouth study which evaluated a $6 \% \mathrm{HP}$ enhanced by titanium dioxide nanoparticles activated by hybrid light (laser/LED) showed delta $\mathrm{E}(\triangle \mathrm{E})$ changes higher than 5 for the $6 \%$ group over three appointments with a total contact time of 72 minutes. ${ }^{26}$ However, this was significantly lower than the control group using 36\% HP. ${ }^{27}$ 


\section{RATIONALE}

A crucial policy change in 2011 resulted in The European Commission issuing a new directive (Council Directive 2011/84/EU) regarding tooth whitening products, classifying these as cosmetics products. Formerly classifying them as medical devices under the Medical Device Directive (MDD 93/42/EEC). The new directive also refers to restrictions and conditions of use of HP in teeth bleaching products -

"Only tooth whitening products with less than 0.1\% HP may be sold on the open market in the European Union and products containing between 0.1\%-6\% HP content may only be sold to dentists, and only under the following conditions: a. The first use of each cycle of the product must be completed by a dentist at a clinical examination, after which use may be continued by the patient, and $b$. The use of these products by clients under 18 years of age is not permitted, even under the supervision of a dentist". ${ }^{28}$

Before this directive, the variety of dental bleaching products and protocols were assured of success with power bleaching treatments, as long as a high concentration HP is incorporated into their system or technique. ${ }^{28}$ The use of a $6 \% \mathrm{HP}$ solution will not be able to match the efficiency of a $35 \% \mathrm{HP}$ solution typically required in a power bleaching treatment.

The decrease in concentration will result in an exponential decrease in efficacy. ${ }^{10}$ To match the same outcome as a higher concentration peroxide multiple and lengthier applications of lower concentration, HP will be required. ${ }^{10}$ Under these conditions, the in-surgery bleaching technique will not be viable, and finding an alternative to enhance the bleaching efficacy of a $6 \% \mathrm{HP}$ solution will be required.

Heating of the bleaching solution is capable of potentially being the enhancement required to reach the required efficacy. From the Arrhenius equation, each $10^{\circ} \mathrm{C}$ increment in temperature increases the rate of reaction 2.2 times, resulting in the increased release of hydroxyl radicals from peroxide. ${ }^{10}$ The heating of the HP may also lead to increased diffusion of HP into the pulp as well as overheating pulp; Zach and Cohen demonstrated that an increase of a mere $5.5^{\circ} \mathrm{C}$ in the pulp tissue temperature could cause irreversible pulpal damage..$^{11,12}$

The use of a bleaching gel has shown to provide an insulating effect for heat transfer to pulp tissue. ${ }^{12,13}$ A 2006 study by Sulieman et al. recorded surface and pulpal temperatures with and without a bleaching agent and after, irradiation for 30 seconds using a diode laser at power settings of $1 \mathrm{~W}, 2 \mathrm{~W}$ or $3 \mathrm{~W}$ and was able to show that the presence of the bleaching agent with photoabsorbers reduced the temperature rise at all power settings by $86-88 \%$. However, they only recommended the $2 \mathrm{~W}$ setting as being safe and being within the parameters described by Zach and Cohen. ${ }^{12}$

In another study, Baldissara et al. were able to demonstrate that increase in pulpal temperatures between $8.9^{\circ} \mathrm{C}$ and $14.7^{\circ} \mathrm{C}$ in humans showed no clinical or histological signs of pulpal damage14. Based on these two studies' findings, the use of a diode laser at $3 \mathrm{~W}$ with 30 s irradiation or a dose of $90 \mathrm{~J} / \mathrm{cm} 2$ can be considered as safe and to not cause pulpal damage.

We need to investigate the possibility of a $6 \% \mathrm{HP}$ solution enhanced with $90 \mathrm{~J} / \mathrm{cm} 2$ of laser energy being able to match the efficiency of a $35 \% \mathrm{HP}$ solution. This study investigates the possibility of a $6 \% \mathrm{HP}$ solution enhanced with $90 \mathrm{~J} / \mathrm{cm} 2$ of laser energy being able to match the efficiency of a $35 \%$ HP solution.

\section{Cartesian shade differentiation}

The CIE system, made up of the tristimulus values $(X, Y$ and Z), on a Cartesian Plane, is the basis of colour language and the way we communicate accurate and precise colour values globally. The representation of RGB or primary colours is by values on the $X, Y$ and $Z$ axes. These $X Y Z$ coordinates are useful in colour matching and colour identity, that is, whether two colours match, but are not, in the calculation of colour difference. The difference between two sets of $X Y Z$ tristimulus values does not yield an accurate numerical value as a representation of this difference. Numerous colour-difference formulae emerged worldwide for a variety of fields and applications. In response to the need for global uniformity, the Commission Internationale de l'Éclairage (CIE), an international authority on light and colour, adopted the CIELAB system in $1976 .{ }^{30}$

The colour difference formula adopted by CIELAB is a technique of converting the $X Y Z$ tristimulus values to produce a precise colour difference value. The $L^{*} a^{*} b^{*}$ system organises all existing colours within a three-dimensional colour space. $L^{*}$ represents the degree of lightness and ranges from 0 (black) to 100 (white); $a^{*}$ represents the green-red axis, while $b^{*}$ represents the blue-yellow axis. The colour comparison before and after treatment computed by the differences between the two colours $(\Delta E)$, which is calculated using the formula:

$\Delta \mathrm{E}=\sqrt{ }\left(\left(\Delta \mathrm{L}^{\star}\right)^{2}+\left(\Delta \mathrm{a}^{\star}\right)^{2}+\left(\Delta \mathrm{b}^{\star}\right)^{2}\right)$

CIELAB is entirely dependent on spectrophotometric measurements of colour; it cannot be used routinely without a colour measurement device, and there is no standard CIELAB colour atlas available. ${ }^{30}$ An instrumental method for shade matching has been preferred over the visual evaluation because it improves the process practically and has statistically higher reliability. ${ }^{31}$

A spectrophotometer is an analytical instrument that is used to quantitatively measure the reflection or transmission properties of a material as a function of wavelength. The VITA Easyshade V (VITA Zahnfabrik, Bad Säckingen, Germany) is a contemporary portable spectrophotometer was used in this study.

The Easyshade $V$ handpiece has a $5 \mathrm{~mm}$ diameter probe, which contains three spectrometers. One spectrometer continuously monitors the output of the lamp during the calibration and measurement process. The other two spectrometers analyse the light that is internally scattered (not absorbed) by the tooth structure. ${ }^{32}$ 
Several factors could affect clinical performance of the intraoral spectrophotometer, positioning on tooth, translucency variations caused by background lighting as well as variations in operator techniques.

\section{AIMS}

This study aims to investigate if a laser enhanced 6\% HP solution is equivalent to a $35 \% \mathrm{HP}$ solution over a two-visit power bleaching treatment protocol, by aiming to prove the following superiority research question:

HO - Null hypothesis: The in-office vital 6\% HP (Group 1/experimental), $A C$ tooth whitening treatment protocol with a diode laser $(810 \mathrm{~nm})$ does not cause colorimetric changes compared with the gold standard 35\% HP (Group 2 -Control) bleaching.

$\mathrm{H} 1$ - Experimental hypothesis: The in-office vital 6\% HP, $A C$ tooth whitening treatment protocol with a diode laser $(810 \mathrm{~nm})$ causes colorimetric changes compared with the gold standard 35\% HP.

\section{MATERIALS AND METHODS}

This clinical study was approved by the Biomedical Research Ethics Committee (BREC) of the University of KwaZulu-Natal (BFC673/18). The study took place between April-May 2019 at a private dental clinic. Fortyseven volunteers were selected and informed consent was obtained.

The bleaching study was a randomised, double-blinded (patients and evaluator) clinical trial with a two-arm parallel-group design without any adaptation in randomisation or conduct to proof a superiority question. The patients were invited to participate in the study through social media advertising, the demographics of which were determined beforehand. Respondents directed to an online questionnaire as the first phase of screening for eligible participants. The final phase of screening involved a physical examination conducted by the primary investigator.

A total of 83 patients were examined to check if they met the following eligibility criteria of the study: older than 18 years of age, good general and oral health, as well as anterior teeth without restorations, previous bleaching procedures, cervical lesions, or dental pain.

Pregnant or lactating patients, gingival recession, periodontal disease, smokers or had other oral-staining habits, tetracycline stains, had moderate or severe fluorosis, orofacial tumours, trauma, orthodontic treatment, or tooth malformation, were taking analgesic, anti-inflammatory, or antibiotic drugs, or if they exhibited high anxiety were excluded.

Four patients were excluded and forty-seven patients were admitted to the study. Informed consent was obtained. Two trained operators (registered dentists) performed the bleaching treatments. The operators were aware of the treatment protocol for each patient. A study coordinator was responsible for patient appointment liaisons, conducting the randomisation, and maintaining, storing and transmitting trial data to the statistician.
Group allocations were performed by random drawing, using Microsoft Excel 2016, from coding assigned to each participant. Group 1 was the experimental group, treated with $6 \%$ hydrogen peroxide (HP6) activated by diode laser. Group 2 acted as a control, receiving the standard 35\% hydrogen peroxide (HP35) treatment.

\section{Sample size calculation}

Calculation of the sample size was done by using nQuery 7.0. Previous studies showed that application of in-office power bleaching agent of 35\% HP HP35 would lead to a $(\triangle E)$ value of $7.0 \pm 2.0$ after two bleaching sessions. ${ }^{25}$ Two study groups each received different treatments, and a continuous primary endpoint was measured.

The primary outcome of this study was efficacy determined by colour alteration $(\Delta \mathrm{E})$. To have an $80 \%$ chance of detecting significance at the level of $5 \%$, considering an increase in the primary outcome measure from $\Delta 7$ in the control group to $\Delta 5$ in the experimental group, a minimum of seventeen participants were required in each group. Allowance for a dropout rate of $23 \%$ and the total number of participants $(n=34)$ was set to forty-four (Computation: test with homogeneous variance nQuery 7.0, procedure MTTO).

\section{Materials}

- For shade measurement - VITA Easyshade V spectrophotometer, S/N H54885 (VITA Zahnfabrik, Bad Säckingen, Germany).

- Activated charcoal powder (Sigma-Aldrich, St Louis, USA).

- Hydrogen peroxide liquid (Sigma-Aldrich, St Louis, USA) in 35\% and 6\% solution stored in dark bottles.

- Elexxion Pico 5W / 810 nm wavelength Diode Laser (Elexxion, Radolfzell, Germany).

\section{Bleaching protocol}

For each bleaching session, the operator mixed the relevant $\mathrm{HP}$ solution with $\mathrm{AC}$ in a glass dappen dish by incorporating $0.1 \mathrm{~g}$ of the $\mathrm{AC}$ powder per $0.5 \mathrm{ml}$ of the HP liquid, namely 6\% HP for Group 1 and 35\% HP for Group 2.

Standard patient protection, as well as a gingival barrier, was applied to the dry gingiva. The gel mixture was then applied to the enamel surface of the upper and lower six anterior teeth to produce a uniform layer approximately $2 \mathrm{~mm}$ thick.

In both groups, the mixture was applied for 20 min, then washed off and a fresh mix of gel placed for another 20 min. In Group 1, however, there was concurrent laseractivation of the gel mixture, cycled twice per each 20 minute application. The handpiece of the diode laser was placed just above the surface of the gel, and applied with a $1 \mathrm{~cm}$ spot diameter for 30 seconds per tooth, on a $3 \mathrm{~W}$ continuous setting. Completion of two bleaching sessions per patient gave a total contact time of 80 minutes.

The interval between sessions was seven days. The re- 
levant bleaching solutions were monitored for uniformity by assigned bleaching staff. The bleach timings and laser application timings (where relevant), were also monitored.

\section{Shade evaluation}

A dedicated Shade Evaluator employing a strict shade evaluation protocol carried out four evaluations per patient in a separate evaluation room. The evaluator was unaware of the protocol used and only saw the assigned patient number ( $\mathrm{n} 1$ to $\mathrm{n} 43$ ) when recording the data.

For both pre-operative evaluations (seven days apart) polishing off the teeth was completed before shade measurement. The two post-operative evaluations were carried out immediately on completion of the bleaching sessions.

\section{Steps in the treatment of patients}

The following steps were followed:

- Shade evaluated with the Vita Easyshade V spectrophotometer.

- Rubber stent formed using Betasil $\circledast$ Vario Putty with a $5 \mathrm{~mm}$ window in stent - window on upper left central incisor, centre of tooth; stent on labial aspect with bite stabilising the stent.

- Vita Easyshade calibrated on cradle (calibration block).

- Average base shade setting chosen.

- Probe placed in window of stabilised stent with the probe window sitting flush against the tooth surface.

- When the device held in a stable position, evaluator took single reading - checked - retook - checked - until two corresponding results were obtained. Thereafter three consecutive readings were taken while held in the same position; the device then gave the average of these five readings. If the first two readings did not correspond then the device was reset, recalibrated and probe positioning was adjusted.

- All the data was manually recorded on patient data sheet.

- The above procedure was repeated for all pre- and post-operative measurements using the same stent (per patient) for all procedures.

\section{Concomitant care}

Patients were urged to adhere to the following between bleaching visits. Items to be avoided: whitening toothpaste, medications which affect saliva flow, mouthwashes, smoking, consumption of chewing tobacco and other items that may cause oral staining such as "paan" (betel leaf) and "supari" (betel nut). Patients also had to limit consumption of tea, coffee and red wine, patients should not have had any other bleaching or dental care in the days between the two appointments.

All participants were monitored by treating dentists, as well as the shade evaluator. Every participant confirmed full compliance. The interventional phase of this clinical trial spanned three weeks with the total time of participant involvement being approximately one month. Overall, the completed processes from inception to the conclusion took the better part of a year.

\section{Statistical methods}

The primary focus is on the description of the mean $\Delta \mathrm{E}$ between the two arms of the study. For the main study, the data for $L^{*}, a^{*}, b^{*}$ and age, as well as the $\Delta E$, are described in terms of means and standard deviations, statistical measures only will describe the data of the pilot study.

The overall $\Delta \mathrm{E}$, calculated as mean $\Delta \mathrm{E}$ over the duration treatment, is used to answer the primary research question by a t-test to evaluate group differences at the $5 \%$ significance level.

To evaluate differences in $\Delta \mathrm{E}, \Delta \mathrm{L}^{*}, \Delta \mathrm{a}^{*}$ and $\Delta \mathrm{b}^{*}$ by visit, age, sensitivity and corresponding interactions of visit by age, was computed by the fitting of linear mixed effects models to the data. Statistical computations were conducted using SAS software; in particular, the application of PROC MIXED. A significance level of $5 \%$ was set.

\section{RESULTS}

The sample for the main study consisted of thirty-two women (82.05\%) and seven men (17.95\%). Nineteen participants were allocated to the experimental arm of the study (Group 1), and twenty to the control arm (Group 2).

\section{Baseline characteristics}

\section{Age and gender distribution}

Age distribution appears to be similar between groups. Mean age in Group 1 was around 30 years (SD 8.7) and in Group 2 around 32 years (SD 9.35).

The gender distribution was as follows - Group 1 consisted of $78.95 \%$ (15) females and 21.05\% (4) males; Group 2 consisted of $85 \%$ (17) females and 15\% (3) males.

\section{Primary analysis $\Delta \mathrm{E}$}

Description of mean $\Delta E$ across visits amounts by 3.4 (SD 1.8) in Group 1 and by 4.9 (SD 3.2) in Group 2. The mean difference between groups was 1.5 (SD 2.6) with a 95\% confidence interval (95\% CL: $-0.19 ; 3.23)$ does not indicate a significant difference at the $5 \%$ level ( $p=0.0798)$.

\section{Sensitivity analysis $\Delta \mathrm{E}$}

We do observe a significant dependency of Age on the $\Delta E(p=0.0041)$ using an averaged 0.1484 increase in $\Delta E$ per patient-year. We do not observe a dependency by group, gender, sensitivity, nor a difference between visits (Tables 1-3).

\begin{tabular}{|l|c|c|c|c|c|}
\hline \multicolumn{4}{|c|}{ Table 1. Description of mean $\Delta \mathrm{E}$ across visits between groups. } \\
\hline Questions & Method & Mean & $95 \%$ CL Mean & Std Dev \\
\hline $\mathbf{1}$ & & 3.3637 & 2.4825 & 4.2449 & 1.8282 \\
\hline $\mathbf{2}$ & & 4.8838 & 3.3788 & 6.3888 & 3.2156 \\
\hline Diff (1-2) & Pooled & -1.5201 & -3.2296 & 0.1894 & 2.6336 \\
\hline
\end{tabular}

\begin{tabular}{|c|c|c|c|c|}
\hline Method & Variances & DF & t Value & $\operatorname{Pr}>|t|$ \\
\hline Pooled & Equal & 37 & -1.80 & 0.0798 \\
\hline
\end{tabular}




\begin{tabular}{|c|c|c|c|c|}
\hline \multicolumn{5}{|c|}{ Type 3 - Tests of fixed effects } \\
\hline Effect & Num DF & Den DF & F Value & $\operatorname{Pr}>\mathrm{F}$ \\
\hline Group & 1 & 33.2 & 0.12 & 0.7293 \\
\hline Visit & 1 & 39.3 & 1.77 & 0.1908 \\
\hline Visit ${ }^{\star}$ Group & 1 & 38 & 1.67 & 0.2040 \\
\hline Gender & 1 & 23.7 & 1.56 & 0.2238 \\
\hline Age & 1 & 42.1 & 9.24 & 0.0041 \\
\hline Age $^{*}$ Group & 1 & 42.8 & 0.01 & 0.9277 \\
\hline Sens & 1 & 30.4 & 1.06 & 0.3109 \\
\hline
\end{tabular}

Sensitivity analysis: L* measurements

We do not observe any influence of age on the $L^{*}$ measurements, as well as differences between gender, visit as well as treatment groups.

\section{Sensitivity analysis: $a^{*}$ and $b^{*}$ measurements}

We do not observe any influence of age on the $a^{*}$ and $b^{\star}$ measurements, as well as differences between gender, visit as well as treatment groups.

\section{DISCUSSION}

In this randomised double-blinded clinical study, a treatment protocol was devised that could effectively measure the energy transfer from the laser light to the bleaching solution and directly correlate this heat transfer to impro- ved efficacy. The addition of heat being the primary mechanistic means of increasing the rate of absorption of a low concentration HP solution rather than directly catalysing the HP solution to release free radicals implicated in the bleaching process.

The lack of similar studies makes it difficult to compare results, however Bortallato et al., (2015), in a parallel study for in-office tooth bleaching with 6\% $\mathrm{H} 2 \mathrm{O} 2 / \mathrm{TiO}$ nanoparticles enhanced by an LED/laser system, showed almost identical $\triangle \mathrm{E}$ values to this study for both the $6 \% \mathrm{HP}$ and $35 \%$ HP groups. ${ }^{24}$ The authors assigned the additional bleaching effect to the photocatalyst of the $6 \%$ nanosized hydrogen peroxide (TiO_N).

Of interest to note in this study, the bleaching gels were additionally coloured, and the combination laser/led device was applied with an intensity of $300 \mathrm{~mW} / \mathrm{cm}^{2}$ for 6 minutes, delivering a dose of $108 \mathrm{~J} / \mathrm{cm}^{2}$ compared to a dose of $90 \mathrm{~J} / \mathrm{cm} 2$ in our study.

Using identical products and parameters as the above study but in a split-mouth study design, Martin et al., $(2015)^{25}$ evaluated 6\% HP enhanced by titanium dioxide nanoparticles activated by hybrid light (laser/LED) showing delta $E(\triangle E)$ changes at visit 2 expressed by mean (standard deviation) similar to this study.

This study was also designed to use a non-commercial

HP35 vs. HP6

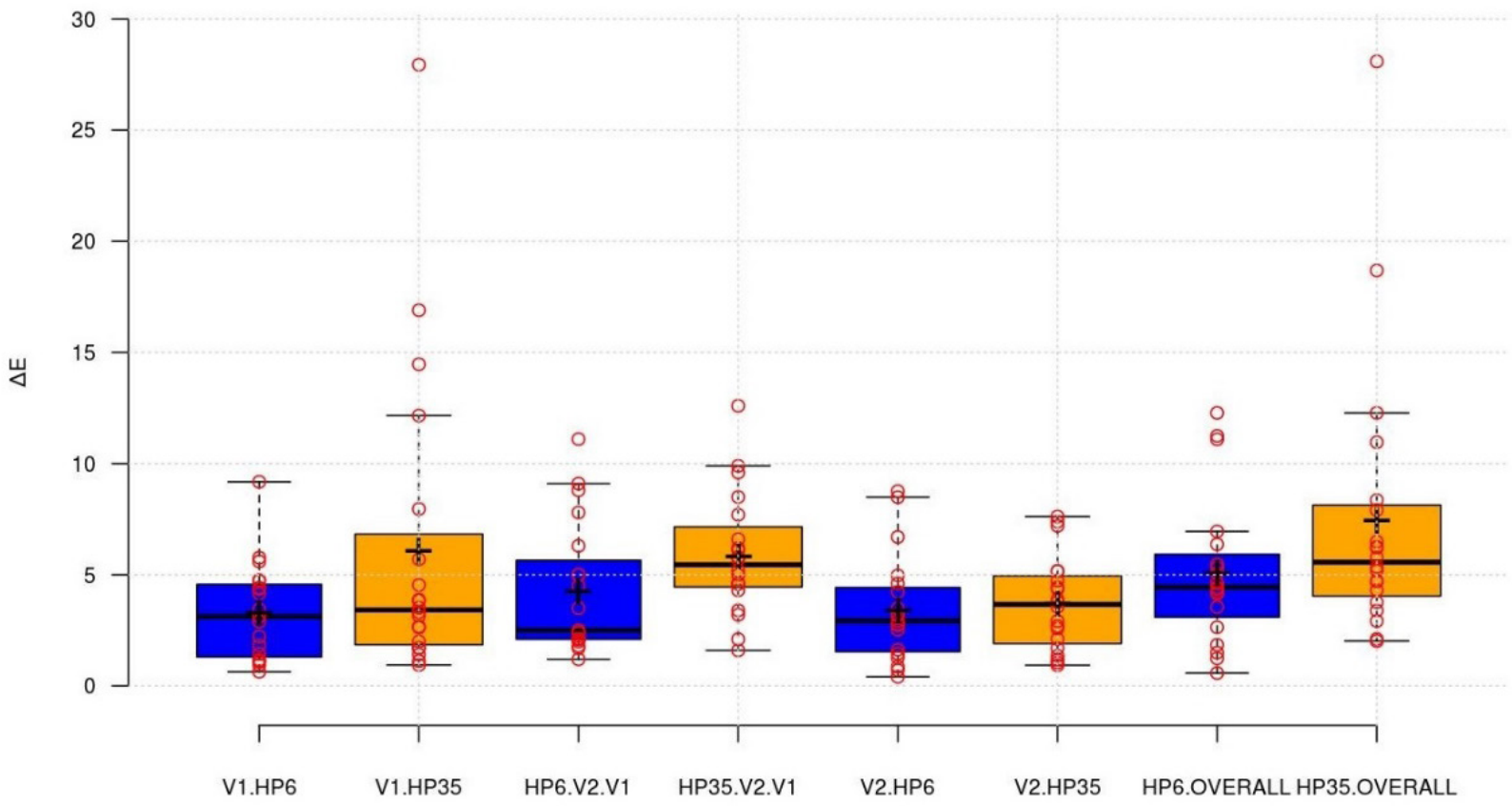

Figure 2. Nature of new cases received 1 July 2020 - 30 September 2020.

\begin{tabular}{|c|c|c|c|c|c|c|c|c|}
\hline \multicolumn{9}{|c|}{ Box plot statistics } \\
\hline & V1.HP6 & V1.HP35 & HP6.V2.V1 & HP35.V2.V1 & V2.HP6 & V2.HP35 & HP6.OVERALL & HP35. OVERALL \\
\hline Upper whisker & 9.18 & 12.16 & 9.10 & 9.90 & 8.49 & 7.52 & 6.95 & 12.28 \\
\hline 3rd quartile & 4.57 & 6.83 & 5.65 & 7.15 & 4.43 & 4.95 & 5.92 & 8.13 \\
\hline Median & 3.12 & 3.42 & 2.50 & 5.45 & 2.93 & 3.67 & 4.44 & 5.56 \\
\hline 1st quartile & 1.30 & 1.85 & 2.10 & 4.45 & 1.54 & 1.90 & 3.09 & 4.04 \\
\hline Lower whisker & 0.64 & 0.94 & 1.20 & 1.60 & 0.41 & 0.93 & 0.58 & 2.02 \\
\hline Nr. of data points & 19.00 & 20.00 & 19.00 & 20.00 & 19.00 & 20.00 & 19.00 & 20.00 \\
\hline Mean & 3.31 & 6.08 & 4.28 & 5.87 & 3.42 & 3.69 & 5.10 & 7.47 \\
\hline
\end{tabular}


bleaching solution devoid of hygroscopic or humectant effects present in commercial bleaching gels. The absence of humectant in our HP solution may account for the lower efficacy results in comparison to the majority of other studies using commercial high concentration HP gels. The gels can dehydrate the tooth, making them appear whiter. The humectant effect of these gels may also account for the improved efficacy associated with bleaching lamps rather than a direct photocatalytic effect.

Humectants have polar hydroxyl groups and can attract water via the weak hydrogen bonds of the latter; by the process of adsorption, they can attract water from their surroundings. Humectants are sensitive to temperature and humidity in the surrounding air when humidity is, high, it can draw water from the air, but in dry air, it will draw moisture from the tooth. Even though some studies have suggested that some of the initial lighter colour changes may be due to dehydration. ${ }^{10,33,34}$ There has been no suggestion, in previous studies, of bleaching lamps enhancing hygroscopic effects of bleaching gels nor of the responsibility of humectants in bleaching efficacy as well as tooth sensitivity.

We cannot correlate $\Delta \mathrm{E}$ changes from one study to another - the methodology of obtaining the $\Delta \mathrm{E}$ needs to be similar, as well as consideration of the baseline data.

\section{Clinical interpretation of data}

A boxplot of $\Delta \mathrm{E}$ data for both groups at the various points was incorporated to further interpret data array (Figure 1 and Table 4).

\section{Median vs. mean analysis}

The boxplot results show us a closer association between the two groups. Of particular interest was the difference in medians being only $1.12 \triangle \mathrm{E}$ units in the overall results (HP35 OVERALL - HP6 OVERALL) showing a much closer association between the two groups than that shown by the difference in means $2.37 \Delta \mathrm{E}$ units.

We may be able to argue reasonably, from looking at the data sets, and the presence of significant singular outliers in the HP35 group, that an analysis of the medians rather than the means may provide a closer estimate of the difference. The median analysis also shows lower differences of the other $\Delta \mathrm{E}$ scores after visit 1 and visit 2 in comparison to the mean analysis for these visits. We cannot conclude or categorically confirm this effect. The "outlier" would increase the SD. As can be seen in Figure 1, the SDs are somewhat different. However, the deviation is in the range of random variation.

\section{Continuity of bleaching post application}

The study also sought to assess the possibility that the teeth continued to whiten after the removal of bleach this would further elucidate the absorption before the activation mechanism of bleaching.

To accomplish this, we measured the difference of $\Delta \mathrm{E}$ between the end of visit one and the start of visit two for both groups. Both groups adequately displayed the effect of continued bleaching, and mean results were comparatively close. However, in terms of medians, the HP35 group was almost twice that of the HP6 group, an expected result, considering the exponential effect of higher concentration on bleaching efficacy.

During the bleaching process of this study, we accelerated the absorption of low concentration HP with heat from the light. The HP, in turn, reacts with organic components within the tooth, which catalyse the breakdown of HP. The subsequent oxidation of the dental phosphoprotein results in whiter teeth.

If we consider the number of organic components readily available to interact with $\mathrm{HP}$ as being limited within a fixed timeframe and consider concentration gradients from the DEJ, then we can theorise a self-limiting effect to bleaching inherent within the tooth. The use of low concentration HP will then be equivalent to a high concentration $\mathrm{HP}$ as long as the absorption rate is the same since the bleaching effect is self-limited by inherent factors within the tooth. However, once absorption stops, the residual higher concentration HP can bleach further with time as organic catalyst becomes available or replenished. This model can account for the first quartile of the 35\% HP group reaching a high $\Delta E$ score of 4.45 , compared to the 2.10 score in the $6 \%$ group.

\section{Results analysis}

The analysis reveals that, concerning the primary endpoint delta $\mathrm{E}$ and the statistical model used according to the sample size argument, the null hypothesis could not be rejected. The result is inconclusive. We did not observe a significant difference between 6\% HP and 35\% HP bleaching groups.

\section{CONCLUSIONS}

Dental bleaching is a dynamic process, which is dependent or not only the breakdown of hydrogen peroxide but also the interaction between the tooth and the composition of the hydrogen peroxide solution. A common feature for all practitioners undertaking bleaching is the unpredictability of results. To date, the elucidation for such unpredictability cannot be reasoned by any particular model. The observations of this study, however, further expounds the idea of an absorption enhancement mechanism rather than a free radical activation as the technique for improving bleaching outcomes.

\section{Study outcome}

In this study, we were not able to demonstrate that the means difference between the two groups at a 95\% confidence interval shows a significant difference at the $5 \%$ level $(p=0.0798)$.

The null hypothesis that the in-office vital 6\% HP, AC tooth whitening treatment protocol with a diode laser $(810 \mathrm{~nm})$ does not cause colourimetric changes compared with the gold standard 35\% HP bleaching cannot be rejected. There is no statistical difference between the two groups, on the mean $\triangle \mathrm{E}$.

This does not infer that both groups are equivalent con- 
cerning $\triangle \mathrm{E}$. The study is inconclusive in this direction. The primary aim in power bleaching is to achieve a maximum whitening effect in the space of a single dental appointment, an idea earlier developed in our introduction. In terms of $\Delta \mathrm{E}$, we could then correlate this to a maximum $\triangle \mathrm{E}$ obtainable in a single visit.

When we consider the highly significant grouping in terms of $\Delta \mathrm{E}$, that is, the lowest performers (first quartile), we see that the most significant improvement in $\Delta \mathrm{E}$ occurs post-bleaching, with the higher concentration performing better. It is, for this reason, we can conclude that low concentration $6 \%$ hydrogen peroxide, even when accelerated with laser light, is not equivalent to a standard high concentration of $35 \%$ hydrogen peroxide solution.

\section{Shortcomings of study}

CIELAB was used as a colour differentiation formula however CIEDE 2000 brings colour differences closer to what the human eye actually perceives and is able to give a more accurate colour difference when used in dentistry. ${ }^{16,17}$

Visual shade guide data an important tool in perceptual shade differentiation has not been include in this study. Further work is required investigating the possible role of humectants in dental bleaching.

\section{Conflict of interest/Competing interests}

The authors declare no conflict of interest or competing interests. This study was funded by the principal researcher.

\section{References}

1. Sulieman M. An Overview of Bleaching Techniques: 3. In-Surgery or Power Bleaching. Dent Update. 2005; 32(2): 101-8. doi:10.12968/denu.2005.32.2.101.

2. Ubaldini ALM, Baesso ML, Medina Neto A, Sato F, Bento AC, Pascotto RC. Hydrogen Peroxide Diffusion Dynamics in Dental Tissues. J Dent Res. 2013; 92(7): 661-5. doi:10.1177/002 2034513488893.

3. Jiang T, Guo YR, Feng XW, et al. Hydrogen Peroxide Might Bleach Natural Dentin by Oxidizing Phosphoprotein. J Dent Res. June 2018:002203451878426. doi:10.1177/002203451 8784260.

4. Bowles WH, Ugwuneri Z. Pulp chamber penetration by hydrogen peroxide following vital bleaching procedures. J Endod. 1987; 13: 375-7.
5. Kwon SR, Wertz PW. Review of the mechanism of tooth whitening. J Esthet Restor Dent. 2015; 27(5): 240-57. doi:10. 1111/jerd.12152.

6. Llena C, Martínez-Galdón O, Forner L, Gimeno-Mallench L, Rodríguez-Lozano FJ, Gambini J. Hydrogen Peroxide Diffusion through Enamel and Dentin. Mater (Basel, Switzerland). 2018;11(9). doi:10.3390/ma11091694.

7. Torres CRG, Wiegand A, Sener B, Attin T. Influence of chemical activation of a $35 \%$ hydrogen peroxide bleaching gel on its penetration and efficacy - In vitro study. J Dent. 2010; 38(10): 838-46. doi:10.1016/J.JDENT.2010.07.002.

8. Camps J, Pommel L, Aubut V, About I. Influence of acid etching on hydrogen peroxide diffusion through human dentin. Am J Dent. 2010; 23(3): 168-70. http://www.ncbi.nlm.nih.gov/ pubmed/20718215. Accessed March 21, 2019.

9. Sulieman M, Addy M, MacDonald E, Rees JS. The effect of hydrogen peroxide concentration on the outcome of tooth whitening: an in vitro study. J Dent. 2004; 32(4): 295-9. doi:10.1016/J.JDENT.2004.01.003.

10. RA Basson, SR Grobler, TJ vW Kotze, Y Osman. Guidelines for the Selection of Tooth Whitening Products amongst Those Available on the Market. www.sada.co.za. Accessed October 28, 2018.

11. Rocha Gomes Torres Av Eng Francisco José Longo C, Maciel Braga Pereira S, Martins Maia Godoy M, Bühler Borges A, Rogério Pucci C, Rocha Gomes Torres C. Influence of Temperature on Bleaching ... Pereira SM et Al Influence of Temperature on the Bleaching Efficacy of Hydrogen Peroxide. Vol 10. https://pdfs.semanticscholar.org/8437/87a24b68c214 3649a1c7270479cfc12cced9.pdf. Accessed March 23, 2019.

12. Zach L, Cohen G. Pulp response to externally applied heat. Oral Surg Oral Med Oral Pathol. 1965;19:515-530. http:// www.ncbi.nlm.nih.gov/pubmed/14263662. Accessed March 24, 2019.

13. Baik JW, Rueggeberg FA, Liewehr FR. Effect of light-enhanced bleaching on in vitro surface and intrapulpal temperature rise. J Esthet Restor Dent. 2001; 13(6): 370-8. http://www.ncbi. nlm.nih.gov/pubmed/11778856. Accessed July 23, 2018.

14. Baldissara P, Catapano S, Scotti R. Clinical and histological evaluation of thermal injury thresholds in human teeth: a preliminary study. J Oral Rehabil. 1997; 24(11): 791-801. http://www.ncbi.nlm.nih.gov/pubmed/9426160. Accessed March 24, 2019.

15. Bruce MacEvoy. handprint: modern color models. https:// www.handprint.com/HP/WCL/color7.html\#CIELAB. Accessed June 17, 2019.

16. Pecho OE, Ghinea R, Alessandretti R, Pérez MM, Della Bona A. Visual and instrumental shade matching using CIELAB and CIEDE2000 color difference formulas. Dent Mater. 2016;3 2(1): 82-92. doi:10.1016/j.dental.2015.10.015.

17. Kim H-K. Evaluation of the repeatability and matching accuracy between two identical intraoral spectrophotometers: an in vivo and in vitro study. J Adv Prosthodont. 2018; 10(3): 252-8. doi:10.4047/jap.2018.10.3.252.

\section{Do the CPD questionnaire on page 105}

The Continuous Professional Development (CPD) section provides for twenty general questions and five ethics questions. The section provides members with a valuable source of CPD points whilst also achieving the objective of CPD, to assure continuing education. The importance of continuing professional development should not be underestimated, it is a career-long obligation for practicing professionals.

\section{Online CPD in 6 Easy Steps}

Go to the SADA website www.sada.co.za.

Log into the 'member only' section with your unique SADA username and password.

Select the CPD navigation tab.

Select the questionnaire that you wish to complete.

Enter your multiple choice answers. Please note that you have two attempts to obtain at least $70 \%$

View and print your CPD certificate. 